\title{
Preparing of the Patient, Performing Diagnostic Flexible Bronchoscopy and Sampling from the Airways
}

\author{
L. Zuccatosta
}

Monaldi Arch Chest Dis 2011; 75: 1, 32-38.

Keywords: Bronchoscopy, Anaesthesia, Sedation, Central lesions, Sampling techniques.

SOD Pneumologia, Azienda Ospedali Riuniti, Ancona, Italy.

Correspondence: Dott.ssa Lina Zuccatosta, SOD di Pneumologia, Azienda Ospedaliero Universitaria “Ospedali Riuniti”, Via Conca 71, 60010 Ancona, Italy; e-mail: linazuccatosta@tiscali.it

\section{Preparing the patient}

The preparation of the patient is a crucial time as it can contribute to the success of the bronchoscopic examination. First and foremost, preparation provides the patient with correct information regarding the procedure by which bronchoscopy is performed and informs him/her of sensations that may be felt. The anxiety and worry manifested by the majority of patients undergoing bronchoscopic examination can in a large part be alleviated by the behaviour of the operators, who must reassure the patient and put them at ease [1] (Level of evidence: III).

Bronchoscopy is carried out with the patient fasting, in order to reduce the volume and acidity of the stomach contents, thus avoiding the risk of possible regurgitation and aspiration of gastric material. A minimum of eight hours' fasting before the bronchoscopy is carried out is recommended [2] (Level of evidence: $I V$ ). The Guidelines of the British Society recommend four hours' fasting [3]. It does not in fact appear that a relatively long fasting time is associated with a lower risk of regurgitation. A meta-analysis [4] of 38 randomised controlled studies on different protocols for pre-operative preparation and post-operative complications found that there is no evidence that a reduction in fasting times, with liquids being taken, is associated with a greater risk of aspiration-regurgitation (Level of evidence: Ia). Drugs for chronic disorders (e.g. anti-hypertensive drugs, digitalis) can therefore be taken normally, with a very small quantity of water.

\section{Premedication}

The rationale for premedication with anticholinergics (atropine, glycopyrrolate) is based on the anti-sialagogue effect which these drugs have, i.e. the inhibition of the vasovagal reflexes (antibradycardia activity) and of bronchoconstriction. No real usefulness however has been demonstrated for their routine use, to offset their potential risks (tachyarrhythmia, acute glaucoma, water retention) (Level of evidence: $I b$ ). In a randomised double-blinded study [5], 217 patients undergoing bronchoscopy with sedation (midazolam) were examined, assigned respectively to the atropine $(0.01$ $\mathrm{mg} / \mathrm{kg})$, glycopyrrolate $(0.005 \mathrm{mg} / \mathrm{kg})$ and placebo groups. No significant difference was observed in the two pre-medicated groups compared with the control group as regards the control of secretions, the amount of anaesthetic used, the incidence of complications or tolerance of the procedure. Similar results were reported in another study [6] in which 55 randomised patients $(25$ pre-medicated with atropine and 30 in the control group) underwent bronchoscopy with Holter-ECG monitoring for 24 hours starting from the beginning of the examination, and no significant difference was found between the two groups as regards the onset of arrhythmias or bradycardia. In addition, no difference was observed between the two groups as regards the extent and control of bronchial secretions (Level of evidence: $I b$ ).

\section{Sedation}

Although the routine use of sedation is not still considered a prerequisite for performing a bronchoscopy, the literature suggests its use [7]. The rationale for sedation is to reduce the state of anxiety, to induce a degree of amnesia but at the same time to keep the patient conscious, capable of collaborating, breathing spontaneously and with adequate ventilation and oxygenation.

Good tolerance of the bronchoscopy on the part of the patient translates into greater facility for the endoscopist in performing the procedure; in addition, the degree of amnesia induced by the sedation makes it easier to perform any subsequent bronchoscopy [8]. The effects of the administration of propofol was evaluated in 18 patients subjected to bronchoscopy under local anaesthesia, observing better tolerance of the examination in the sedated group, while in the control group, be- 
sides greater discomfort, an increase was reported in cardiac frequency and systemic arterial pressure [9]. No significant differences, however, were observed regarding the reduction of oxy-haemoglobin saturation during the examination (Level of evidence: $I b)$. In another study [10] the cardiovascular effects consequent on sedation with propofol and midazolam in 100 patients undergoing bronchoscopy were compared, and better control of pressure values and cardiac frequency in the group sedated with propofol was observed, also reporting good tolerance by patients with pre-existing pulmonary disease (Level of evidence: $I b$ ). Good results were obtained with the use of benzodiazepine. A randomised study [11] on 100 patients undergoing bronchoscopy, proved better tolerance in patients treated with diazepam compared with those not treated, without significant cardiovascular changes (episodes of desaturation did not differ significantly between the two groups) (Level of evidence: $I b$ ). Midazolam (soluble benzodiazepine) is one of the most widely-used drugs because of the manageability it provides in relation to the brief duration of its action (two hour half-life) and its good sedative and amnesic action. Satisfactory sedation is obtained at a dosage of $2.5 \mathrm{mg}$ in the majority of patients [12]. In a survey on performing bronchoscopy in England and Wales, midazolam appeared to be the drug of choice in $85 \%$ of cases [13] (Level of evidence: IV).

The use of opioids seems to ensure better control of coughing, but it does not affect comfort and tolerance of the examination in terms of reduction of anxiety [14]. The combination of opioids and benzodiazepine has been reported to be effective in controlling coughing, especially when invasive procedures are planned. Good results [15] have been reported with the combination midazolamhydrocodone (120 randomised patients, subjected to sedation with midazolam and opiate vs midazolam alone). The patients treated with the combination had better control of coughing and better tolerance of the examination compared with the group treated with midazolam alone, without significant changes between the two groups as regards saturation (Level of evidence: $I b$ ).

\section{Recommendations}

- The explanatory interview (on how and why the bronchoscopy is being carried out) prepares and reassures the patient in terms of the procedure and facilitates tolerance (Grade C).

- A fasting period of $\mathbf{4}$ hours before the examination is sufficient (Grade B).

- The treatment usually given (antihypertensive drugs, etc.) can be taken by mouth with water, even in the four hours preceding the procedure (Grade $\mathrm{A})$.

- A peripheral vein must always be incannulated, irrespective of whether sedative drugs are used, in order to have a rapid access route in the event that it should become necessary to administer drugs during the course of the procedure or at the end (Grade $\mathrm{C}$ ).

- Premedication with atropine does not significantly reduce the risk of cardiac complications and does not offer benefits in the control of secretions. Its routine use is therefore not justified (Grade B).

- Sedation improves tolerance of the procedure on the part of the patient (control of anxiety and of the haemodynamic parameters) and facilitates its execution; it should be used except in the case of obvious contraindications (Grade B).

\section{References}

1. Poi PJH, Chuah SY Liam CK. Common fears of patients undergoing bronchoscopy. Eur Respir J 1998; 11: 1147-1149.

2. Reed AP. Preparation of the patient for awake flexible bronchoscopy. Chest 1992; 101: 244-253

3. British Thoracic Society Bronchoscopy Guidelines Committee, a Subcommittee of Standards of Care Committee of British Thoracic Society. British Thoracic Society guidelines on diagnostic flexible bronchoscopy. Thorax 2001; 56 Suppl 1: i1-21.

4. Brady M, Kinn S, Stuart P. Preoperative fasting for adults to prevent perioperative complications. Cochrane Database Syst Rev 2003; (4): CD004423.

5. Cowl CT, Prakash UB, Kruger BR. The role of anticholinergics in bronchoscopy. A randomized clinical trial. Chest 2000; 118: 188-192.

6. Grahmann PR, Schoder A, Warzelhan J, et al. Bronchoscopy and rhythmic disorders. Premedication with atropine-sulphate, as a rule? Pneumologie 2002; 56: 593-598.

7. Matot I, Kramer MR. Sedation in outpatient bronchoscopy. Respir Med 2000; 94: 1145-53.

8. Maguire GP, Rubinfeld AR, Trembath PW, Pai MC. Patients prefer sedation for fiberoptic bronchoscopy. Respirology 1998; 3: 81-85.

9. Gonzales R, De La Rosa Ramirez I, Maldonado-Hernan$\operatorname{dez} \mathrm{A}$, et al. Should patients undergoing a bronchoscopy be sedated? Acta Anaesthesiol Scand 2003; 47: 411-41515.

10. Oztuk T, Cakan A, Guerce G, et al. Sedation for fiberoptic bronchoscopy: fewer adverse cardiovascular effects with propofol than with midazolam. Anasthesiol Intensiv Med Notfallmed Schmerzther 2004; 39: 597-602.

11. Putinati S, Ballerin L, Corbetta L, et al. Patient satisfaction with conscious sedation for bronchoscopy. Chest 1999; 115: 1437-1440.

12. Crawford M, Pollock J, Anderson K, et al. Comparison of midazolam with propofol for sedation in outpatient bronchoscopy. Br J Anaesth 1993; 70: 419-422.

13. Pickles J, Jeffrey M, Datta A, et al. Is preparation for bronchoscopy optimal? Eur Respir J 2003; 22: 203-206.

14. Houghton CM, Raghuram A, Sullivan PJ, et al. Premedication for bronchoscopy: a randomized double blind trial comparing anfetanil with midazolam. Respir Med 2004; 98: 1102-1107.

15. Stolz D, Chhjed PN, Leuppi JD, et al. Cough suppression during flexible bronchoscopy using combined sedation with midazolam and hydrocodone: a randomized, double blind, placebo controlled trial. Thorax 2004; 59: 773-776. 


\section{Local anaesthesia}

Lidocaine is the most widely-used substance as a topical anaesthetic in the respiratory tree and is available in aqueous solution (1, 2 or $4 \%$ ), gel $(4 \%)$, unguent $(5 \%)$, and metered spray $(10 \%, 10$ $\mathrm{mg}$ per spray). The duration of the action is about 15-20 minutes, and absorption is slower in the upper respiratory tracts (nose, pharynx, larynx) compared with the tracheobronchial tree (which has a larger surface area). Plasmatic concentration is consequently greater when instilled into the lower airways. Since lidocaine is metabolised in the liver, it must be administered with caution in patients with liver diseases and used carefully in patients with low cardiac ejection (depression of myocardial contractility and arrest) [1, 2]. In a study [3] the seric concentration of lidocaine was measured in 16 patients undergoing bronchoscopy, without evidence of adverse effects within a dosage of 500 mg (Level of evidence: IIa). In a randomised study [4] carried out on 96 patients undergoing bronchoscopy, equal efficacy (control of coughing, tolerance of the examination and need for further administration during the examination) was encountered in the use of lidocaine at $1 \%$ and at $2 \%$, no adverse effect being observed for a maximum plasmatic concentration of 5.02 and $6.28 \mathrm{mcg} / \mathrm{ml}$ (Level of evidence: $\mathrm{Ib}$ ). In a study carried out on 51 asthmatic patients subjected to bronchoscopy [5], toxicity was not observed for a maximum lidocaine dosage of $600 \mathrm{mg}$, equivalent to 8.2 $\mathrm{mg} / \mathrm{kg}$ (Level of evidence: IIa). In patients with liver or cardiac diseases a recommendation was made not to exceed a dose of $5 \mathrm{mg} / \mathrm{kg}[6,7]$. Administration methods include nebulisation, instillation through the bronchoscope and transcricoidal injection (the latter is currently rarely used as it is less comfortable than the other methods). It has been demonstrated [8] that administration of lidocaine by nebulisation before bronchoscopy reduces the necessity for further administration by the endobronchial route, lowering the risk of toxicity (the plasmatic level is dose-dependent) (Level of evidence: IIa). Different conclusions were however drawn by another prospective randomised study [9], in which the average dosage of lidocaine was higher in the group of patients subjected to pre-examination nebulisation than in the placebo group, demonstrating furthermore that nebulisation had no effect in the reduction of coughing or in improving tolerance of the examination (Level of evidence: $I b$ ).

The use of lidocaine in gel form seems better than the spray formulation in topical anaesthesia of the nose. The spray formulation and gel were compared [10], and a lower degree of pain was reported in the group of patients anaesthetised with gel (Level of evidence: $I b$ ). Similar conclusions were drawn when evaluating three different methods of topical nasal anaesthesia (gel, spray and tampons moistened with lidocaine in solution), and it was reported that the use of gel was the least irritating procedure and the one preferred by patients [11] (Level of evidence: $I b$ ).

\section{Recommendations}

- The maximum dose of lidocaine in the execution of a bronchoscopy should be limited to $8.2 \mathrm{mg} / \mathrm{kg}$ of bodyweight, with the total dosage anyway not exceeding $600 \mathrm{mg}$ (Grade B).

- In patients with liver or cardiac diseases the dosage should not exceed 5 $\mathrm{mg} / \mathrm{kg}$ of body-weight (Grade B).

- Nebulisation of lidocaine does not improve tolerance of the examination nor reduce the total dose of anaesthetic used (Grade A).

- Lidocaine in a gel preparation is preferable for nasal anaesthesia (Grade A).

\section{References}

1. Foldes FF, Molloy R, McNall PG, et al. Comparison of toxicity of intravenously given local anaesthetic agents in man. JAMA 1969; 72: 1493-1498.

2. Shelley MP, Wilson JP, Norman J. Sedation for fiberoptic bronchoscopy. Thorax 1989; 44: 769-775.

3. Milman N, Laub M, Munch, et al. Serum concentrations of lignocaine and its metabolite monoethylglycinexylidide during fiberoptic bronchoscopy in local anesthesia. Respir Med 1998; 92: 40-43.

4. Mainland PA, Kong AS, Chung DC, et al. Adsorption of lidocaine during aspiration anesthesia of the airway. J Clin Anesth 2001; 13: 440-446.

5. Langmack EL, Richard J, Martin MD, et al. Serum lidocaine concentration in asthmatics undergoing research bronchoscopy. Chest 2000; 117: 1055-1060.

6. Efthimiou J, Higenbottam T, Holt D, et al. Plasma concentrations of lignocaine during fiberoptic bronchoscopy. Thorax 1982; 37: 68-71.

7. British Thoracic Society Bronchoscopy Guidelines Committee, a Subcommittee of Standards of Care Committee of British Thoracic Society. British Thoracic Society guidelines on diagnostic flexible bronchoscopy. Thorax 2001; 56 Suppl 1: i1-21.

8. Foster WM, Hurewitz AN. Aerosolized lidocaine reduces dose of topical anesthetic for bronchoscopy. Am Rev Respir Dis 1992; 146: 520-522.

9. Stolz D, Prashant N, Chhajerd MD, et al. Nebulized lidocaine for flexible bronchoscopy. Chest 2005; 128: 1756-1760.

10. Zainudin BM, Rafia MH, Sufarlan AW. Topical nasal anaesthesia for flexible fiberoptic bronchoscopy: lignocaine spray or gel? Singapore Med J 1993; 34: 148-149.

11. Randell T, Yli-Hankala A, Valli H, et al. Topical anaesthesia of the nasal mucosa for fiberoptic airway endoscopy. Br J Anaesth 1992; 68: 164-167.

\section{Performing fiberoptic bronchoscopy}

Fiberoptic bronchoscopy is a procedure carried out under local anaesthetic with the patient seated or lying down. The choice of position for the patient and for the performance of the examination (from the front or from behind) depend on the operator's habitual practice; if the examination is performed with the patient seated, a chair must be 
used which is readily reclinable if the necessity arises. The bronchoscope may be introduced nasally, orally or by tracheostomy.

The choice of the nasal or oral route depends also on the experience of the operator [1,2]. Some authors suggest the nasal route because it offers a more direct anatomical path (which is preferable when positioning a tracheal tube), a more stable position for the instrument (useful in the case of transbronchial needle aspiration, use of lasers or argon plasma coagulation), and better control of secretions from the oral cavity. Conversely, the oral route is preferable in the case of congenital or post-surgical anomalies, removal of foreign bodies and in the case of severe disorders of the coagulation with haemorrhagic diathesis (possible nosebleeds). There are no studies which recommend the choice of one route rather than the other ( Level of evidence: $I V$ ).

\section{Recommendations}

- Fibre-optic bronchoscopy can be carried out either with the patient seated or with the patient supine. In the event that the examination is being performed with the patient seated, it is necessary to use a chair which is readily reclinable (Grade $\mathrm{C}$ ).

- Use of the nasal route is preferable when carrying out bronchoscopy; the oral route should be reserved for cases of occlusion or restriction of the naval cavities, in the event of disturbances to coagulation or when there is a suspected foreign body (Grade C).

\section{References}

1. Metha AC, Dweik RA. Nasal versus oral insertion of flexible bronchoscope. Pro nasal insertion. J Bronchol 1996; 3: 224-228.

2. Guntupalli KK, Siddiqi AJ. Nasal versus oral insertion of flexible bronchoscope. Pro oral insertion. J Bronchol 1996; 3: 229-233.

\section{Administration of oxygen}

The appearance of hypoxemia is a fairly frequent phenomenon in the course of a bronchoscopy and may persist for some hours after the procedure. The causes which induce a reduction in partial oxygen pressure $\left(\mathrm{PaO}_{2}\right)$ include excessive sedation, suction manoeuvres which remove oxygen and reduce pulmonary volumes below the residual functional capacity, bronchospasm, and alveolar collapse caused by bleeding or by instillation of fluids [1, 2]. Hypoxemia can give rise to arrhythmias and it is therefore important to maintain during the examination a degree of saturation which allows the examination to be carried out without incurring this risk. An oxy-haemoglobin saturation value of $90 \%$ has been indicated as a minimum level for carrying out a bronchoscopy in safety, with constant monitoring of the patient by pulse oxymeter. In a randomised study on 160 patients subjected to bronchoscopy [3], the patients were divided into four groups of which the first had no oxygen administered, and the others were administered respectively $2 \mathrm{l} / \mathrm{min}$ by nasal prongs, $21 /$ min by pharyngeal catheter and $31 /$ min by pharyngeal catheter. Group 1 (without oxygen) manifested oxy-haemoglobin saturation of $85 \%$ with incidence of tachycardia and bradycardia in $20 \%$ of cases, while group 4 (oxygen at $3 \mathrm{l} / \mathrm{min}$ ) maintained a saturation of $94 \%$ with tachycardia and bradycardia in $10 \%$ of cases. The administration of oxygen reduced the incidence of arrhythmias by $50 \%$ (Level of evicence $I b$ ). Discordant opinions are reported in the literature as regards the routine administration of oxygen during bronchoscopy (in patients who present a basal saturation level above $90 \%$ ). In 1,051 patients subjected to bronchoscopy without supplemental administration of oxygen before the procedure and with known $\mathrm{FEV}_{1}$ [4], administration of oxygen became necessary during or at the end of the procedure in only 151 patients (14\%), while 101 patients $(9.6 \%)$ had transient desaturation without the need for correction with oxygen. A significant correlation was furthermore observed between $\mathrm{FEV}_{1}$ value and desaturation (the lower $\mathrm{FEV}_{1}$ value, the greater was the need for oxygen), while sedation (midazolam) did not influence the probability of the occurrence of desaturation. In this study supplemental oxygen was administered in $35 \%$ of patients with $\mathrm{FEV}_{1}$ below 1 litre, in $14 \%$ of patients with $\mathrm{FEV}_{1}$ of 1-1.5 l, and only in $7 \%$ of cases with $\mathrm{FEV}_{1}$ above 1.51 . On the basis of these results, the authors concluded that desaturation can occur in the course of bronchoscopy for any value of $\mathrm{FEV}_{1}$ and irrespective of the sedation used, but the majority of patients do not require the administration of oxygen, especially those who have an $\mathrm{FEV}_{1}$ greater than 1.51 ( Level of evidence: III). Similar results but different considerations were reported in another study on 44 patients [5] who did not include anyone with a $\mathrm{PaO}_{2}$ below $65 \mathrm{mmHg}$, known cardiovascular illnesses or risk factors for cardiovascular disease. It was observed that many patients showed desaturation independently of the use of sedation and with normal $\mathrm{PaO}_{2}$ values before the bronchoscopy. The most important factor which correlates with desaturation is the presence of an obstructive component, but since $\mathrm{FEV}_{1}$ is not capable of predicting the possibility of the appearance of desaturation, and since desaturation can appear for any value of $\mathrm{FEV}_{1}$ even in patients with normal arterial saturation of oxygen, the administration of oxygen should be carried out routinely during bronchoscopy (Level of evidence: IV). Patients considered at risk for hypoxemia in the course of bronchoscopy are elderly subjects (>80) with pulmonary fibrosis. In a study on 336 elderly patients subjected to bronchoscopy [7], an incidence of desaturation of $55 \%$ was observed in patients with fibrosis, but only in $16 \%$ of cases the administration 
of oxygen was necessary (Level of evidence: III). In a randomised study on 97 patients subjected to bronchoscopy there were no significant differences in terms of $\mathrm{SaO}_{2}$ between delivering oxygen with nasal prongs and by cannula through the mouth (Level of evidence: $\mathrm{Ib}$ ). In a randomised study conducted on 34 patients subjected to bronchoscopy with sedation (diazepam), patients with altered pulmonary function in whom the oxygen was suspended immediately after the procedure had episodes of desaturation not pointed out in the group in which oxygen was maintained for the two subsequent hours. The authors suggest maintaining the administration of oxygen for two hours in sedated patients and in those with functional alterations $\left(\mathrm{FEV}_{1}<75 \%\right.$ of predicted value), until the initial values are recovered [8] (Level of evidence: $I b)$.

\section{Recommendations}

- All patients must be monitored by pulse oxymeter during bronchoscopy (Grade B).

- The pulse oxymeter must be positioned before the administration of sedatives (Grade C).

- The administration of oxygen during bronchoscopy is obligatory in patients with basal $\mathrm{SaO}_{2}$ lower than $\mathbf{9 0 \%}$, in cases of desaturation $(<90 \%)$ and in patients with cardiac diseases (risk of arrhythmia) (Grade B).

- In patients with basal saturation levels $>\mathbf{9 0 \%}$ the administration of oxygen is not recommended as routine (Grade B).

- The administration of oxygen must be kept up with monitoring by pulse oxymeter for 1-2 hours after the end of the bronchoscopy in patients with cardiac diseases, in those with altered pulmonary function, and in the event that profound sedation becomes necessary (Grade B).

\section{References}

1. Dubrawsky C, Awe RJ, Jenkins DE. The effect of bronchofiberscopic examination on oxygenation status. Chest 1975; 67: 137-140.

2. Brach BB, Escano GG, Harrell JH, et al. Ventilationperfusion alterations induced by fiberoptic bronchoscopy. Chest 1976; 69: 335-337.

3. Milman N, Fauershon P, et al. Pulse oxymetry during fiberoptic bronchoscopy in local anesthesia: frequency of hypoxemia and effect of oxygen supplementation. Respiration 1994; 61: 342-347.

4. Jones AM, O'Driscol R. Do all patients require supplemental oxygen during flexible bronchoscopy? Chest 2001; 119: 1906-1909.

5. Golpe R, Mateos A. Supplemental oxygen during flexible bronchoscopy. Chest 2002; 121: 663-664.

6. Shinagawa N, Yamazaki K, Kinoshita I, et al. Suscep- tibility to oxygen desaturation during bronchoscopy in elderly patients with fibrosis. Respiration 2006; 73: 90-4.

7. McCain TW, Dunagan DP, Adair NE, et al. Prospective randomized trial comparing oxygen administration during nasal flexible bronchoscopy: oral vs nasal delivery. Chest 2001; 120: 1671-1674.

8. Kristensen M, Milman N, Jarving I. Pulse oxymetry at fiberoptic bronchoscopy in local anaesthesia: indication for postbronchoscopy oxygen supplementation? Respir Med 1998; 92: 432-437.

\section{Bronchial washing, brushing, bronchial biopsy}

The principal sampling methods used to take samples of endoscopically visible bronchial lesions are bronchial washing, brushing and biopsy.

Bronchial washing is a simple sampling technique and consists of instilling $20 \mathrm{ml}$ of sterile saline solution into the bronchial tree, which is recovered and sent to the laboratory for cytological, immunological and microbiological tests. Brushing is a cytological sampling technique in which special small brushes are introduced into the working channel of the bronchoscope; the material retrieved is smeared on a glass slide and fixed in ethyl alcohol. Single-use brushes are preferable to the reusable type, diagnostic yield being equal, because of the risk of possible contamination and cross-infection [1]. Bronchial biopsy provides histology tissue specimens, but the material sampled can also be used for a cytological analysis, thus increasing the diagnostic yield, smearing the tissue fragment on glass [2] or immersing the biopsy fragments in rinse solution before transferring them to formalin [3].

The number of biopsy samples reported in the literature as necessary for a good diagnostic yield in lung cancer varies from three to six in central lesions $[4,5]$. A study on 271 patients, of whom 215 had endoscopically visible tumours [4] demonstrated a diagnostic yield of $65.2 \%$ with a single biopsy sample, while five biopsies were necessary for reaching a sensitivity of $90 \%$ (Level of evidence: III). The BTS guidelines recommend five biopsy samples where there is a suspicion of neoplastic lesion [6].

There are many reports in literature concerning the use and sensitivity of the three sampling methods, both evaluated individually and in combination, in the diagnosis of lung cancer. Sensitivity is reported [7] for bronchial washing, brushing and biopsy in central lesions of 27-90\%, 44-94\% and $51-97 \%$ respectively, while in peripheral lesions the sensitivity of brushing is $48 \%$, and that of bronchial washing much lower at $28 \%$. Brushing provides a better diagnostic yield in cases of infiltrative lesions, while biopsy, which has good sensitivity in vegetative lesions, shows a lower diagnostic yield in the infiltrative forms and in those with a large necrotic component.

In a meta-analytic study [8] concerning the sensitivity of the various sampling techniques in suspected neoplastic lesions, the best yield in central lesions is given by bronchial biopsy (74\%), 
then by brushing (59\%) and by bronchial washing $(43 \%)$; overall sensitivity of the three methods combined is $88 \%$ (Level of evidence: IIa).

In a study conducted on 98 patients with central lesion [9], a sensitivity of $82 \%$ is reported for biopsy, $68.4 \%$ for brushing and $31.6 \%$ for bronchial washing. The combined use of biopsy and brushing increases sensitivity for malignancy to $88 \%$, while no increase in sensitivity is found with the combination of bronchial biopsy and bronchial washing. A low diagnostic yield is reported for bronchial washing $(22.7 \%)$ in peripheral neoplastic lesions [10]. In 56 patients affected by central neoplastic lesions and subjected to biopsy, brushing and washing, the sensitivity of the methods was respectively $95.5 \%, 79.5 \%$ and $56.8 \%$. Brushing was the sole diagnostic means in $4.5 \%$ of the cases, while in no cases did bronchial washing alone allow diagnosis [11]. Similar results are reported in another study [12], which demonstrates a minimal alteration in sensitivity for malignancy in central lesions when biopsy $(85.3 \%)$ is combined with washing and brushing $(88.1 \%)$. In a retrospective analysis [13], bronchial washing was the sole diagnostic procedure in central neoplastic lesions in 6 out of 222 patients (2.7\%), with a greater sensitivity in lesions with a necrotic component, and low probability of diagnostic yield in bleeding lesions. The use of bronchial washing in addition to biopsy and brushing in central lesions does not have an acceptable cost/efficacy ratio, and its use may find a rationale only in selected cases, such as neoplastic lesions with a large necrotic component or in case of negativity of biopsies and brushing. The combined use of biopsy and brushing was considered [14] the best combination in the diagnosis of central neoplastic lesions (sensitivity 78\%), while washing has an extremely low diagnostic yield. An evaluation was performed recently of the role of bronchial washing carried out before and after biopsy and brushing, in terms of diagnostic yield and cost, in patients with suspected lung cancer [15]. The global diagnostic yield was $76 \%$ and $36 \%$ respectively for central and peripheral lesions when washing was carried out before biopsy and brushing, and $74 \%$ and $42 \%$ when washing was carried out after biopsy and brushing. In $6 \%$ of cases washing was the only component which allowed the diagnosis of malignancy. No significant differences were demonstrated in the diagnostic yield of washing carried out before and after sampling with forceps or brush. The authors conclude that although the additional diagnostic yield offered by washing and by brushing is relatively low, the cost/benefit ratio justifies their use in patients with suspected lung cancer.

On the basis of these studies it is evident that the best sensitivity in central lesions is obtained with biopsy forceps (Level of evidence: III), and that the combination of biopsy and brushing increases the diagnostic yield significantly (Level of evidence: III). Bronchial washing considered as a stand-alone procedure has a low sensitivity and little impact on altering sensitivity when it is com- bined with the other sampling methods (Level of evidence: III).

\section{Recommendations}

- In cases of central bronchogenic lesion the biopsy forceps must be considered as the sampling instrument of first choice (Grade B).

- In central lesions a minimum of three biopsy samples are required for a good diagnostic yield (Grade B); five samples are recommended for a high probability of diagnosis of malignancy (Grade B).

- The combination of bronchial biopsy and brushing can be used to increase the diagnostic yield in central lesions, especially in case of an infiltrative pattern (Grade B).

- The routine use of bronchial washing is not justified, considered as a single procedure or in combination, in the diagnosis of central lesions (Grade B).

\section{References}

1. Kinnear WJM, Wilkinson MJ, James PD, et al. Comparisons of the diagnostic yields of disposable and reusable cytology brushes in fiberoptic bronchoscopy. Thorax 1991; 46: 667-668.

2. Popp W, Rauscher H, Ritschka L, et al. Diagnostic sensitivity of different techniques in the diagnosis of lung tumors with the flexible fiberoptic bronchoscope: comparison of brush biopsy, imprint cytology of forceps biopsy and histology of forceps biopsy. Cancer 1991; 67: 72-75.

3. Rosell A, Monso E, Lores, et al. Cytology of bronchial biopsy rinse fluid to improve the diagnostic yield for lung cancer. Eur Respir J 1998; 12; 1415-1418.

4. Gellert AR, Rudd RM, Sinha G, et al. Effect of multiple bronchial biopsies on the diagnosis yield in bronchial carcinoma. Thorax 1982; 37; 684-687.

5. Shure D, Astarita RW. Bronchogenic carcinoma presenting as an endobronchial mass: optimal number of biopsy specimens for diagnosis. Chest 1983; 83: 865-867.

6. British Thoracic Society Bronchoscopy Guidelines Committee, a Subcommittee of Standards of Care Committee of British Thoracic Society. British Thoracic Society guidelines on diagnostic flexible bronchoscopy. Thorax 2001; 56 Suppl 1: i1-21.

7. Mazzone P, Jain P, Arroliga AC, et al. Bronchoscopy and needle biopsy techniques for diagnosis and staging of lung cancer. Clin Chest Medicine 2002; 23: 137-158.

8. Schreiber G, McCrory D. Performance Characteristics of different modalities for diagnosis of suspected lung cancer. Chest 2003; 123: 115S-128S.

9. Karahalli E, Yilmaz A, Turker H, et al. Usefulness of various diagnostic techniques during fiberoptic bronchoscopy for endoscopically visible lung cancer: should cytology examinations be performed routinely? Respiration 2001; 68: 654-655.

10. Baaklini WA, Reinoso MA, Gorin AB. Diagnostic yield of fiberoptic bronchoscopy in evaluating solitary pulmonary nodules. Chest 2000; 117: 1049-54. 
11. Amorin A, Lombardia E, et al. Lung cancer diagnosis: comparison of post-bronchoscopy sputum cytology, bronchial washing, brushing and biopsy. Rev Port Pneumol 2003; 9: 44-45.

12. Fauzi AR, Balakrishnam L, Rathor MY. Usefulness of cytological specimens from bronchial washings in addition to endobronchial biopsies during bronchoscopy for lung cancer: 3 years data from chest clinic in a general hospital. Med J Malaysia 2003; 58: 729-734.

13. Liwrsisakun $\mathrm{C}$, Pothirat $\mathrm{C}$, Bumroongkit $\mathrm{C}$, et al. Role of bronchial washing in the diagnosis of endoscopical- ly visible lung cancer. J Med Assoc Thai 2004; 87: 600604.

14. Soler TV, Isamitt DD, Carrasco OA. Yield of biopsy, brushing and bronchial washing through fiberbronchoscopy in the diagnosis of lung cancer with visible lesions. Rev Med Chil 2004; 132: 1198-1203.

15. Van der Drift MA, Van der Wilt GJ, Thunnissen FB, et al. A prospective study of the timing and cost-effectiveness of bronchial washing during bronchoscopy for pulmonary malignant tumors. Chest 2005; 128: 16-8.

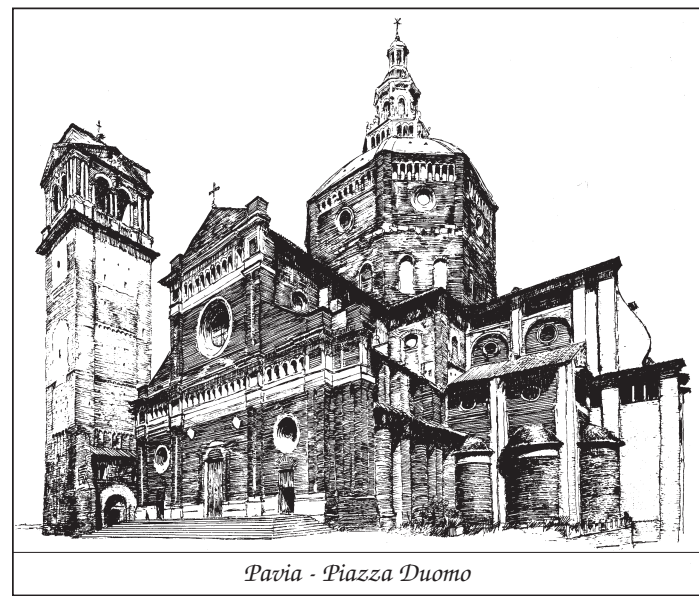

